Gastroenterologe 2009 · 4:191-192

DOI 10.1007/s11377-009-0318-y

Online publiziert: 7. Mai 2009

(c) Springer Medizin Verlag 2009

\author{
M. Fried ${ }^{1} \cdot$ W.E. Schmidt ${ }^{2}$ \\ ${ }^{1}$ Klinik für Gastroenterologie und Hepatologie, Universitätsspital Zürich \\ ${ }^{2}$ I. Medizinische Klinik, Klinikum der Ruhr-Universität-Bochum, \\ St. Josef-Hospital, Bochum
}

\title{
Ösophagus- und Magenkarzinom
}

Die Karzinome des Ösophagus und des Magens gehören zu den häufigsten malignen Tumoren und tragen erheblich zur Karzinommortalität bei. Große Fortschritte in der Diagnostik dieser Tumoren mit einem deutlich verfeinerten Staging sind in den letzten Jahren zu verzeichnen. Dies führte zu einer zunehmend differenzierten, stadiengerechten Therapie. Auch bei der Behandlung dieser Tumoren im lokal fortgeschrittenen Stadium ist es durch Entwicklung multimodaler Therapiestrategien zu großen Fortschritten gekommen. So haben optimierte Verfahren der Chemotherapie und Radiochemotherapie einen signifikanten, positiven Einfluss auf das Überleben der betroffenen Patienten, was neuere Daten belegen. Die endoskopische Therapie wurde in den letzten Jahren weiterentwickelt und belegt einen zunehmend wichtigeren Platz in der Behandlung von Frühformen dieser Tumoren. Neuere operative Verfahren wurden entwickelt und evaluiert mit im Vergleich zu früheren Verfahren deutlich besseren Ergebnissen bei geringerer Morbidität. In diesem Heft werden die neuesten Entwicklungen auf diesem wichtigen Gebiet durch ausgewiesene Experten dargestellt und kritisch für die Anwendung in der ärztlichen Praxis gewichtet.

Neben den bekannten Risikofaktoren wie Alkohol und Rauchen sind heute Umweltfaktoren und das Übergewicht in den Vordergrund getreten. So kann das seltenere Auftreten der Magenkarzinome zumindest teilweise durch veränderte Ernährungsgewohnheiten, insbesondere die seltenere Verwendung von Salz zur Konservierung von Nahrungsmitteln, erklärt werden. Wahrscheinlich haben auch die Eradikation des H. pylori und seine sel- tenere Verbreitung in den westlichen Ländern einen Beitrag zur verminderten Inzidenz des Magenkarzinoms geleistet, obwohl die Datenlage weiterhin nicht eindeutig ist. Bemerkenswert ist der deutliche Anstieg des Adenokarzinoms des Ösophagus im Vergleich zum Plattenepithelkarzinom, der zumindest teilweise durch die Kausalkette Übergewicht und gastroösophagealer Reflux erklärt werden kann.

\section{Optimierte Radiochemo- therapie-Verfahren haben das Überleben signifikant verbessert}

Die neuen, differenzierteren therapeutischen Verfahren benötigen eine entsprechend verfeinerte Diagnostik und standardisierte Staging-Verfahren, die unter anderem auch die Endoskopie mit „narrow band imaging“, die Endosonographie, das Spiral-CT von Thorax und Abdomen und, beim Ösophaguskarzinom und Adenokarzinom des ösophagogastralen Übergangs, die Positronenemissionstomographie (PET oder PET-CT) einschließen. Beim Magenkarzinom kann mit einer Staging-Laparoskopie eine okkulte Peritonealkarzinose ausgeschlossen werden, was für Therapie und Prognose eine entscheidende Bedeutung haben kann. Der Stellenwert neuerer endoskopischer Verfahren, wie die konfokale Laserendomikroskopie und die Endozytoskopie, bleibt unklar und ist Gegenstand von aktuellen Studien.

Die Chemotherapie und die Radiochemotherapie haben heute einen etablierten Platz in der Behandlung des Ösophagus- und des Magenkarzinoms, insbe- sondere als perioperative Therapie beim Magenkarzinom und als präoperative, neoadjuvante Therapie beim Ösophaguskarzinom. So führt die multimodale Therapie bei der Behandlung von lokal fortgeschrittenen Magen- und Ösophaguskarzinomen zu einer Verbesserung der Prognose. Beim Magenkarzinom sind neuere Verfahren, wie monoklonale EGFR-Antikörper, Anti-VEGF-Antikörper oder Catumaxomab, ein intraperitoneal verabreichter Antikörper gegen ein epitheliales Adhäsionsmolekül, Gegenstand von kontrollierten Studien.

Beim Plattenepithelkarzinom hat sich in den tieferen Stadien 1 und 2 die alleinige chirurgische Behandlung, bei den höheren Stadien die neoadjuvante Radiochemotherapie möglichst mit einer transthorakalen En-bloc-Ösophagektomie und Zweifeld-Lymphadenektomie (Abdomen und Mediastinum/Thorax; D2-Lymphknotendissektion, D2-LAD) durchgesetzt. Diese zeigt bessere Ergebnisse als die definitive Radiochemotherapie. Der Stellenwert der minimal-invasiven Ösophagektomie ist noch unklar. Erste Ergebnisse aus amerikanischen und japanischen Zentren sind ermutigend, aber randomisierte Studien fehlen noch.

Bei den Adenokarzinomen des ösophagogastralen Übergangs hat sich die Klassifikation nach Siewert durchgesetzt und dient der optimalen Therapieplanung. Neben den Standardverfahren der transthorakalen En-bloc-Ösophagektomie mit Lymphadenektomie und hoch intrathorakaler Anastomose für den Typ I (BarrettKarzinom) sowie der transhiatal erweiterten Gastrektomie mit Lymphadenektomie im unteren Mediastinum und D2LAD und Rekonstruktion durch supradia- 
phragmale Ösophagojejunostomie RouxY wird in den letzten Jahren bei Frühkarzinomen die limitierte chirurgische Resektion mit gestielter Jejunuminterposition erprobt. Erste Ergebnisse sind vielversprechend.

\section{$>$ Die endoskopische Mukosaresektion kann immer häufiger eine Operation ersparen}

Beim Magenkarzinom ist die totale Gastrektomie nur noch bei Tumoren des oberen und mittleren Drittels und bei Linitis plastica indiziert. Bei distalen Magenkarzinomen wird heute eine 4/5 (sog. subtotale)-Resektion favorisiert. Nach den aufsehenerregenden Resultaten japanischer Chirurgen mit einer standardisierten erweiterten D2-LAD stehen jetzt auch Studien aus westlichen Ländern, die diese aufwendige Technik evaluiert haben, zur Verfügung. Die D2-LAD hat in der holländischen Multicenterstudie gegenüber der eingeschränkten D1-LAD im Stadium IIIA signifikante Vorteile aufzuweisen. Sie ist heute das Verfahren der Wahl in Zentren. Eine Erweiterung der Lymphknotendissektion über das Kompartiment 2 (sog. $\mathrm{D}_{3}$ - oder $\mathrm{D}_{4}$-LAD) hinaus ist aber nach der neuesten randomisierten Studie aus Japan nicht indiziert.

Einen besonderen Stellenwert zur Planung einer limitierten Resektion und
LAD bei Frühkarzinomen könnte neuen Methoden, wie der Kombination von präoperativer radioaktiver Markierung und intraoperativer Farbstoffmarkierung der ersten drainierenden Wächter-Lymphknoten („dual mode sentinel node technique"), zukommen. Auch für das Magenkarzinom sind minimal-invasive Techniken und limitierte Resektionen entwickelt worden. Diese beinhalten zum Beispiel die laparoskopische/offene pyloruserhaltende distale Magenresektion beim Magenfrühkarzinom im unteren Drittel. Diese Techniken sind in Japan entwickelt worden und bedürfen der weiteren Evaluation in westlichen Zentren.

Bemerkenswert sind die Forschritte bei der Behandlung von Frühkarzinomen des Magens und insbesondere des Ösophagus durch eine endoskopische Mukosaresektion, die immer mehr Patienten eine Operation ersparen. Auch hier ist die Datenlage noch begrenzt, und weitere Erfahrungen sind nötig, um die Grenzen der Indikationen und den Langzeitverlauf besser abschätzen zu können. Jedoch haben diese Techniken schon heute einen etablierten Platz in der Behandlung von Frühkarzinomen des Ösophagus und des Magens, sofern sie auf die Mukosa beschränkt sind (sogenannte Tra-Tumoren).

Die Therapie der Ösophagus- und Magenkarzinome hat große Fortschritte gemacht, ist jedoch auch deutlich differenzierter geworden. Diese Komplexität setzt erfahrene interdisziplinäre Teams von spezialisierten Chirurgen, Gastroenterologen, Onkologen und Radioonkologen voraus. Patienten mit diesen Tumorerkrankungen sollten deshalb bevorzugt in spezialisierten Einrichtungen behandelt werden, um ein möglichst optimales Behandlungsergebnis zu erzielen.

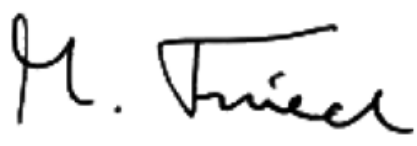

M. Fried

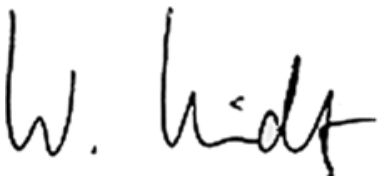

W.E. Schmidt

Korrespondenzadresse

Prof. Dr. M. Fried

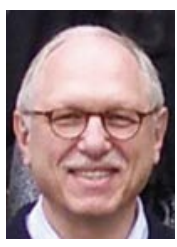

Klinik für Gastroenterologie und Hepatologie, Universitätsspital Zürich Rämistr. 100, 8091 Zürich

Schweiz

michael.fried@usz.ch

\section{Prof. Dr. W.E. Schmidt}

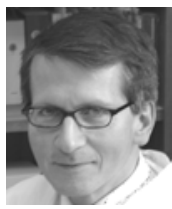

I. Medizinische Klinik, Klinikum der Ruhr-Universität-Bochum, St. Josef-Hospital, Gudrunstr. 56, 44791 Bochum wolfgang.schmidt@ ruhr-universitaet-bochum.de

\section{Exklusiv für Abonnenten und Gesellschaftsmitglieder :}

\section{Nutzen Sie das Online-Archiv von Der Gastroenterologe}

Ihre Vorteile:

- Alle bisher erschienenen Hefte kostenlos online verfügbar

- Ob unterwegs oder am eigenen PC: Zugriff überall und jederzeit

- Komfortable und schnelle Recherche nach Themen, Autoren,

Suchbegriffen

\section{Registrieren Sie sich jetzt unter}

www.DerGastroenterologe.springer.de

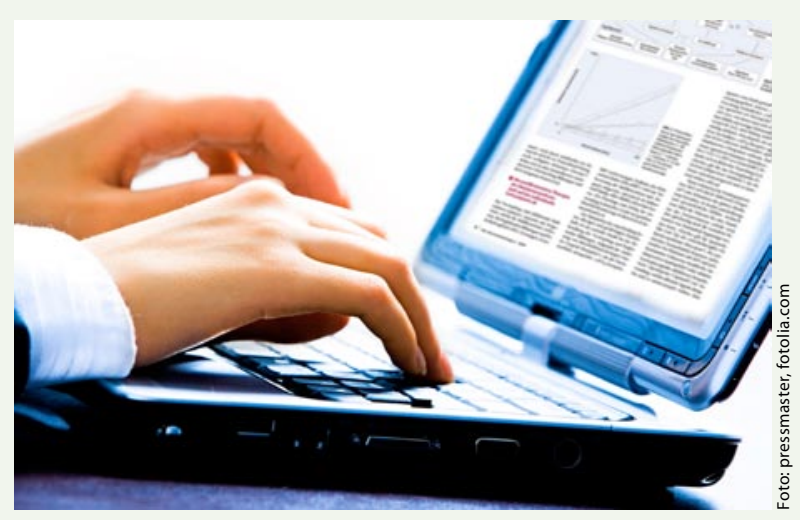

Mitglieder der folgenden Fachgesellschaften können diesen Online-Service kostenlos nutzen:

- Mitteldeutsche Gesellschaft für Gastroenterologie

- Gastroenterologische Arbeitsgemeinschaft Rheinland-Pfalz/Saarland

- Gesellschaft für Gastroenterologie Nordrhein-Westfalen 